\title{
Les sources d'une altérité religieuse en Révolution : Rabaut Saint-Étienne ou la radicalisation des représentations protestantes
}

The sources of a religious "otherness" in Revolution: Rabaut Saint-Étienne or the radicalization of Protestant representations

\section{Céline Borello}

\section{(2) OpenEdition \\ Journals}

\section{Édition électronique}

URL : https://journals.openedition.org/ahrf/13353

DOI : 10.4000/ahrf.13353

ISSN : 1952-403X

\section{Éditeur :}

Armand Colin, Société des études robespierristes

\section{Édition imprimée}

Date de publication : 1 décembre 2014

Pagination : $29-49$

ISBN : 978-2-200-92928-2

ISSN : 0003-4436

\section{Référence électronique}

Céline Borello, «Les sources d'une altérité religieuse en Révolution : Rabaut Saint-Étienne ou la radicalisation des représentations protestantes », Annales historiques de la Révolution française [En ligne], 378 | octobre-décembre 2014, mis en ligne le 01 décembre 2014, consulté le 01 juillet 2021. URL : http://journals.openedition.org/ahrf/13353; DOI : https://doi.org/10.4000/ahrf.13353 


\title{
LES SOURCES D'UNE ALTÉRITÉ RELIGIEUSE EN RÉVOLUTION : RABAUT SAINT-ÉTIENNE OU LA RADICALISATION DES REPRÉSENTATIONS PROTESTANTES
}

Céline BORELLO

\begin{abstract}
Après avoir exercé des fonctions pastorales à Nîmes, Rabaut SaintÉtienne participe à différents travaux et assemblées révolutionnaires à Paris. Durant la décennie 1790, il apparaît dans des caricatures, pamphlets, ouvrages de propagande qui deviennent autant de supports permettant l'expression de fantasmes et de dénigrements. Audelà de l'anecdotique, ceux-ci révèlent ce que ce pasteur représente alors et, à travers lui, le protestantisme. L'enquête propose de suivre comment cet individu, du fait de son appartenance confessionnelle et de ses fonctions dans la Révolution, incarne un bouc émissaire idéal pour détracteurs et partisans de la Révolution.
\end{abstract}

Mots-clés : Rabaut Saint-Étienne, protestantisme, huguenot, radicalisation, pasteur, caricature, calomnie.

« Je sais bien que depuis le commencement de la Révolution, un parti, dont les desseins pernicieux sont trop peu connus, ne néglige rien pour inspirer au Peuple un fanatisme bien plus dangereux que celui qu'il prétend combattre, puisque le levain qui met ce fanatisme en fermentation, est un poison anti-monarchique, dont les funestes effets ne se sont déjà que trop manifestés [...]. Mais il faut désabuser [le] peuple [...], il faut le prévenir que l'affectation avec laquelle les protestants occupent l'Assemblée nationale [...], cache des desseins secrets et des vues sans doute perfides » 
Lettre de M. Boyer à M. Bergasse, Paris, le 15 décembre $1790^{1}$.

En 1989, lors du bicentenaire de la Révolution française, Jacques Poujol fait paraître un long article, fort documenté, intitulé « Le changement d'image des protestants pendant la Révolution ». Voulant répondre à la question du passage « de la quasi-invisibilité des protestants à une notoriété considérée comme scandaleuse », il conclut par « une détérioration systématique de l'image protestante $»^{2}$. Les mots de Boyer semblent confirmer la charge négative de la représentation huguenote. Pourtant, durant les XVII ${ }^{\mathrm{e}}$ et XVIII ${ }^{\mathrm{e}}$ siècles, les réformés ou « nouveaux convertis » n'ont jamais été invisibles et leur notoriété est souvent jugée scandaleuse dans les livres à succès du temps : certains passages du Siècle de Louis XIV de Voltaire, trop rapidement présenté comme le défenseur des huguenots, le révèlent, tout comme l'apologétique catholique ou, au siècle précédent, celle des jansénistes ${ }^{3}$.

Y a-t-il réellement changement d'image des protestants français et détérioration de cette dernière au moment de la Révolution ? Cette réflexion s'appuiera sur Jean-Paul Rabaut dit Rabaut Saint-Étienne (1743-1793), mis en scène par une célèbre image de la Révolution et de l'idéal de Nation rassemblée : le Serment du jeu de Paume de David. Situé au premier plan, pacifiant les religions avec Dom Gerle et l'abbé Grégoire, le pasteur de Nîmes, député de cette ville et de Beaucaire en 1789, puis de l'Aube en 1792, membre de nombreux comités, président de la Constituante et de la Convention, apparaît dans d'autres représentations ou textes des années

(1) Lettre adressée par M. Boyer, substitut du Procureur de la Commune, \& député du Corps Municipal de Nismes à Paris, à M. Bergasse, avocat, Député à l'Assemblée Nationale, Paris, le 15 décembre 1790 , p. 1 et p. 6.

(2) Jacques POUJOL, «Le changement d'image des protestants pendant la Révolution », Les protestants et la Révolution française, Bulletin de la société de l'Histoire du Protestantisme Français, t. 135, 1989, p. 502 et p. 539. « Image » est à considérer ici comme représentation des protestants par ceux qui ne le sont pas, en tant qu'idée donc que s'en font essentiellement les catholiques.

(3) D'après le philosophe, la rébellion protestante reste inscrite dans l'histoire politique du XVIII ${ }^{\mathrm{e}}$ siècle : « Après la mort à jamais effrayante et déplorable de Henri IV, dans la faiblesse d'une minorité et sous une cour divisée, il était bien difficile que l'esprit républicain des réformés n'abusât de ses privilèges, et que la cour, toute faible qu'elle était, ne voulût les restreindre. Les huguenots avaient déjà établi en France des cercles, à l'imitation de l'Allemagne. Les députés de ces cercles étaient souvent séditieux, et il y avait dans le parti des seigneurs pleins d'ambition. Le duc de Bouillon, et surtout le duc de Rohan, le chef le plus accrédité des huguenots, précipitèrent bientôt dans la révolte l'esprit remuant des prédicants et le zèle aveugle des peuples », Voltaire, Euvres complètes, Le Siècle de Louis XIV, volume 4, Paris, A. Ozanne, 1838, p. 183. L'opposition des jansénistes à l'égard du protestantisme s'atténue durant le XVIII' ${ }^{\mathrm{e}}$ siècle et le rôle des premiers dans la quête des droits des seconds est réel (Charles O'BRIEN, « The jansenist campaign for toleration of protestants in late eighteenth century France », Journal of History of Ideas, vol. 46, n ${ }^{\circ}$, 1985, p. 523-538, et « Jansénisme et tolérance civile à la veille de la Révolution », Catherine MAIRE (dir.), Jansénisme et Révolution, Chroniques de Port-Royal, 1990, p. 131-145). 
1789-1799. La dénonciation féroce dont il fait l'objet dans ces sources invite à chercher sur quels griefs et en quoi Rabaut Saint-Étienne paraît un bouc émissaire tout désigné pour symboliser le huguenot. Cependant, évoquer une «détérioration » de l'image protestante paraît excessif, et même erroné dans le contexte politique radicalisé des premières années de la Révolution française.

\section{Dénoncer le huguenot à travers Rabaut Saint-Étienne}

Tentons une classification des dénonciations d'après les sources iconographiques et textuelles de l'époque, essentiellement issues des secteurs catholique, anti-révolutionnaire ou contre-révolutionnaire. Images, pamphlets, ouvrages de propagande, chansons et poèmes révèlent une stigmatisation contre un groupe religieux et plus particulièrement un personnage, érigé en symbole négatif, Rabaut Saint-Étienne.

Sur trois types d'accusation, le premier est d'ordre social. Alors que dans Le serment du Jeu de Paume, une poignée de main est signe d'entente et de paix, d'autres figures donnent de l'action de Rabaut une image plus négative. Une caricature, Les Braves brigands d'Avignon, datée du 16 décembre 1791, démontre l'argument ${ }^{4}$. Comme toutes les autres, cette iconographie contre-révolutionnaire se fonde sur les événements du moment, ici le massacre de la Glacière du 16 octobre $1791^{5}$. Trois personnages sont représentés : Armand-Gaston Camus, député de Paris, Charles-François Bouche, député du Tiers de la sénéchaussée d'Aix, et Rabaut, député de Nîmes. La gravure, violente, exprime la dérégulation sociale avec le ministre protestant qui clame : « Ce joli coup de Rabot me fait venir à la bouche ». Les jeux de mots, dont est friand le XVIII ${ }^{\mathrm{e}}$ siècle, se multiplient en relation avec les patronymes des principaux instigateurs. Ailleurs, dans cette caricature, un groupe se livre à une scène d'anthropophagie avec la légende : "protestants de Nîmes, de la Vaunage et de la

(4) Six cents caricatures révolutionnaires et environ cent cinquante caricatures contrerévolutionnaires (entre novembre 1791 et avril 1792) ont été identifiées par les historiens. Sur la caricature de la période voir les travaux fondateurs de Claude Langlois, Antoine de Baecque ou Annie Duprat : Antoine DE BAECQUE, La caricature révolutionnaire, Paris, Presses du CNRS, 1988 ; Claude LANGLOIS, La caricature contre-révolutionnaire, Paris, Presses du CNRS, 1988 ; Annie DUPRAT, Le Roi décapité : essai sur les imaginaires politiques, Paris, Cerf, 1992 ; id., Histoire de France par la caricature, Paris, Larousse, 2000. La caricature est reproduite en annexe : Caricature 1- Les Braves brigands d'Avignon. Présentée dans Claude LANGLOIS, La caricature contre-révolutionnaire, op.cit., p. 71.

(5) Notons que les seules caricatures du temps qui fassent mention du protestantisme le représentent sous les traits de Rabaut Saint-Étienne. 
Gardonnenque qui se font bonne bouche ». Les trois hommes, accompagnés du patriote Jourdan, protagoniste de l'affaire d'Avignon, marchent sur une foule de cadavres, renforçant la violence de leurs actes. De ces détails des événements méridionaux des années 1790-1791, rappelons simplement qu'en juin 1790 a lieu la «bagarre de Nîmes $»^{6}:$ sur fond d'élection municipale perdue, les protestants nouent des liens avec les patriotes de la Vaunage et de la Gardonnenque. Une altercation entre gardes nationales rivales dégénère alors et provoque de fortes violences urbaines. C'est le camp protestant et patriote qui semble l'emporter. Se déploie en fait, dans la caricature, un mélange des différents événements qui s'échelonnent de mai 1790 à octobre 1791, une sorte de compte rendu de l'action huguenote mais un bilan déformé. Car si, effectivement, dans le Languedoc, les protestants sont impliqués, rien en revanche ne justifie leur présence dans le cas du « massacre d'Avignon ». Le pasteur Rabaut se réjouit de la scène et approuve les déchaînements du Midi qui rappellent un autre temps de brutalité entre catholiques et protestants, celui des guerres de religion du $\mathrm{XVI}^{\mathrm{e}}$ siècle, matrices de violences extrêmes ${ }^{7}$. Rabaut est rendu responsable de celles de la période 1790-1791 dans un grand sud protestant. La mention des événements méridionaux se retrouve également dans une autre caricature, légèrement antérieure et datée du 6 novembre, intitulée Les Coups de Rabot ${ }^{8}$. Parmi les copeaux des coups du pasteur, les massacres de Nîmes apparaissent, tout comme ceux de Montauban et d'Uzès' ${ }^{9}$. Le mot «massacre » ainsi réitéré, renvoie une nouvelle fois

(6) Michel VovelLE, « La place de Nîmes dans les Révolutions méridionales », AHRF, Nîmes au temps des Révolutions, 1789-1848, n² 258, 1984, p. 449-456 ou Burdette C. POLAND, French Protestantism and the French Revolution. A Study in Church and State, Thought and Religion, 16851815, Princeton, Princeton University Press, 1957. Parmi les travaux les plus récents sur le sujet voir Valérie SotTOCASA, Mémoires affrontées, protestants et catholiques face à la Révolution dans les montagnes du Languedoc, Rennes, Presses universitaires de Rennes, 2004, p. 38 et $s q$.

(7) Denis CROUZET, Les guerriers de Dieu. La violence au temps des troubles de religion, vers 1525 - vers 1610, Paris, Champ Vallon, 1994. Claude Langlois note un rapprochement entre la scène d'anthropophagie et la caricature de Gillray, Petit souper à la parisienne, relative aux massacres de Septembre (Claude LANGLOIS, La caricature contre-révolutionnaire, op.cit., p. 72). Sur l'utilisation du mot « massacre » durant la Révolution voir Jean-Clément MARTIN, « Massacres, tueries, exécutions et meurtres de masse pendant la Révolution, quelles grilles d'analyse ? », La Révolution française [En ligne], Les massacres aux temps des Révolutions, mis en ligne le 15 février 2011, URL : http://lrf.revues.org/201.

(8) Se retrouve le jeu de mot sur le nom de Rabaut Saint-Étienne. La caricature est reproduite en annexe : Caricature 2- Les Coups de Rabot. Présentée dans Claude LANGLOIS, La caricature contre-révolutionnaire, op.cit., p. 55.

(9) À Montauban, le 10 mai 1790 une émeute provoque la mort de cinq gardes nationaux et entraîne un exode temporaire de la population protestante (voir Daniel LiGOU, Montauban à la fin de l'Ancien Régime et aux débuts de la Révolution : 1787-1794, Paris, M. Rivière, 1958). À Uzès, en février 1791, des tensions éclatent, fruits des antagonismes des alentours (voir François PUGNIÈRE, Les cultures politiques à Nîmes et dans la Bas-Languedoc oriental du XVIIe siècle aux années 1970 : 
au $\mathrm{XVI}^{\mathrm{e}}$ siècle et l'analogie avec la Saint-Barthélemy opère fortement aux yeux des contemporains ${ }^{10}$. Le souvenir du 24 août 1572 est d'ailleurs réactivé par Mirabeau alors que Rabaut s'exprime à l'Assemblée nationale au moment de la discussion sur l'article 10 de la Déclaration des droits de l'homme et du citoyen, le 23 août $1789^{11}$. La violence demeure constante pour dénoncer le danger potentiel des représailles protestantes.

Un deuxième type d'accusation sort du cadre de la dérégulation sociale pour toucher au politique et pour faire de Rabaut l'homme qui a voulu mettre fin à la monarchie en imposant une république à la France. Ainsi d'autres copeaux des Coups de Rabaut contiennent les expressions «République fédérative » et «Point de roi ». Mais l'idée fonctionne pleinement dans un pamphlet paru à Nîmes, le 20 mars 1790 : Les Républiques fédératives ou lettre de M. Rabaut Dypuy à M. RabautSaint-Etienne son frère ${ }^{12}$. Il est ici question de déplacer, de l'ordre social dérégulé à l'ordre politique renversé, les reproches qui lui sont adressés, et à travers lui, aux huguenots. Rabaut est non seulement responsable du désordre public mais également à la source d'une volonté de destituer le monarque. Sur 64 folios, son frère et son père le louent de faire aboutir les plans secrets des réformés : à maintes reprises de leur histoire, les

Affrontements et dialogues, Paris, L'Harmattan, 2008, p. 125 et sq. D'autres villes méridionales, non mentionnées dans la caricature, sont touchées par les tensions nîmoises à l'exemple d'Arles (voir Pierre SERNA, Antonelle. Aristocrate révolutionnaire 1747-1817, Paris, Éditions du Félin, 1997, p. 139).

(10) Timothy Tackett évoque des rumeurs de « guerre de religion » dans le Midi au moment des affaires de Montauban et Nîmes : Timothy TACKETT, La révolution, l'Église, la France, Paris, Cerf, 1986, p. 341.

Dans le contexte nîmois, un autre massacre est gardé en mémoire et présent dans les pamphlets anti-protestants : la Michelade de 1567 (par exemple dans Le Précis historique des massacres commis par les protestants, op. cit., p. 45).

(11) Les protestants prennent la parole, ce jour-là, autour de la proposition de rédaction de l'article X émanant du comte de Castellane et en réaction à l'amendement proposé par l'abbé Dillon, « Nul ne doit être inquiété pour ses opinions religieuses, pourvu qu'elles ne troublent pas l'ordre public » (voir Nathanaël WEISS, « Les séances des 22 et 23 août 1789 à l'assemblée nationale », Bulletin de la société de l'histoire du protestantisme français, t. 38, 1889, p. 561-575). Parmi eux, Rabaut prononce un discours dans lequel il demande la liberté de conscience et de culte pour tous (Opinion de M. Rabaut de Saint-Étienne sur la motion suivante de M. le Comte de Castellane : "Nul ne doit être inquiété pour ses opinions religieuses, ni troublé dans l'exercice de sa religion », Versailles, Baudoin, 1789). Les débats n'étant pas terminés, et reportés au lendemain 24 août, la prise de parole protestante fait dire à Mirabeau «N'oubliez pas que demain c'est la Saint-Barthélemy » (Jacques PoUJOL, op. cit., p. 525). Voir également Philippe JouTARD, Janine EsTEBE, Élisabeth LABRousse, Jean LeCUIR, La SaintBarthélémy ou les résonances d'un massacre, Neuchâtel, Delachaux et Niestlé, 1976 et David EL KENZ « Le massacre de la Saint-Barthélemy est-il un lieu de mémoire victimaire ? », David EL KENZ, François-Xavier NÉRARD (dir.), Commémorer les victimes en Europe du XVI e siècle à nos jours, Seyssel, Champ Vallon, 2011, p. 217-236.

(12) Les Républiques fédératives. Lettre de M. Rabaut Dupuy à M. Rabaut Saint-Etienne son frère, ministre protestant, député de Nîmes. Membre du comité de constitution et Président de l'Assemblée nationale, Nîmes, mai 1790. 
huguenots auraient fomenté des plans pour diviser la France en « Cantons ou Républiques fédératives, suivant les plans tracés [par nos Auteurs] dans des assemblées tenues à Nisme, à Anduze, à Montauban, à la Rochelle, en $1572,1573,1575,1585$ et $1621 »^{13}$. En 1797, Les Véritables auteurs de la Révolution française réexaminent sur plus de six cents pages les événements de la Révolution au miroir de ceux de la Réforme et placent Rabaut Saint-Étienne au cœur du récit, comme grand chef d'orchestre, avec Necker ${ }^{14}$, de la fin de la monarchie. Et, après lui avoir consacré de longs passages, l'auteur conclut :

« Rabaut consomma les projets régicides que son parti avait ébauché et parvint à anéantir [...] le parti royaliste. Ainsi le premier cri de la Convention, son premier acte fut la République $»^{15}$.

En poursuivant l'inventaire « des méfaits » commis par Rabaut, ses détracteurs veulent montrer que les bouleversements apportés par les protestants ne sont pas seulement politiques, mais également religieux. Ils dénoncent en effet la destruction de la religion, catholique bien entendu. Dans Les Véritables auteurs de la Révolution, le discours de Rabaut du 23 août 1789 est ainsi présenté :

«Leur religion, pour laquelle Rabaud de Saint-Etienne demandait la tolérance, est devenue l'arbitre intolérante du culte catholique; les ministres protestants, pour lesquels Rabaud de Saint-Etienne réclamait la liberté d'exercice, l'ont obtenue ; et les ministres catholiques l'ont perdue. Le culte que Rabaud de Saint-Etienne revendiquait pour sa secte, parce que disait-il, le culte est un dogme, a cessé d'être un dogme pour les catholiques [...]. Les églises catholiques ont été profanées ou détruites ou converties en temple pour l'usage et les prêches des Calvinistes eux-mêmes $»^{16}$.

L'auteur dresse le tableau d'un renversement total de situation religieuse, tout comme le montre encore une troisième caricature, datée

(13) Les Républiques fédératives, op. cit., $\mathrm{f}^{\circ} 4$ (de nombreux folios décrivent par la suite les décisions prises par les huguenots lors de ces réunions). Sur les assemblées politiques protestantes du $\mathrm{XVI}^{\mathrm{e}}$ siècle voir Janine GARRISSON, Protestants du Midi, 1559-1598, Privat, Toulouse, rééd. 1991. Sur les débats autour de l'expression « Provinces unies du Midi » voir Michel PERRONET, « La République des Provinces unies du Midi : les enjeux de l'historiographie »; Anne BLANCHARD, Henri MichEL, Élie PÉLAQUIER (dir.), La vie religieuse dans la France méridionale, Montpellier, Université Paul Valéry, 1992, p. 5-26.

(14) Necker est déjà présent dans Les Républiques fédératives, op.cit., p. 28.

(15) JOURDE [attribué à N. SOURDAT], Les Véritables auteurs de la Révolution de France de 1789, Neuchâtel, 1797, p. 35-36.

(16) Ibidem, p.16-17 
du 29 décembre 1791, où trois personnages constituent la scène, Camus de nouveau - Talleyrand, évêque d'Autun, et Rabaut ${ }^{17}$. Chacun représente un responsable de la fin de la religion catholique, dessinée sous les traits d'une femme voilée : le jansénisme, la philosophie et le protestantisme. Le contexte de la Constitution civile du Clergé est au cœur de cette caricature (le député Camus avait présenté un discours en mai 1790 sur le sujet) ainsi que le décret contre les réfractaires, voté par la Législative le 29 novembre 1791. L'anéantissement de la religion catholique résulterait d'un complot longuement dévoilé lui aussi dans Les véritables auteurs de la Révolution ${ }^{18}$, ou bien encore dans le Secret échappé qui parait en avril $1790^{19}$. Dans ce pseudo-dialogue, Rabaut converse avec deux Anglaises qui l'interrogent sur les événements révolutionnaires. Et, expliquant le rôle de l'assemblée et des protestants pour « ôter à la [religion] catholique sa qualité de dominante dans le royaume », il termine par la confidence suivante :

«si nous nous occupons sérieusement d'améliorer le sort des Protestants en France, et de leur obtenir des privilèges opiniâtrement refusés jusqu'à ce jour à leur religion; au moins est-il bon qu'ils le sachent, afin qu'ils aident l'œuvre commune par leurs efforts particuliers, et que de concert entre nous, il résulte un succès qui mette le comble à nos vœux. Mais je ne voudrois pas, mesdemoiselles, que la fin de notre conversation fût connue des catholiques, qui ne manqueroient pas de sonner le tocsin, comme si leur religion étoit perdue, ou que le feu fût à leurs maisons ».

Avant d'examiner le fondement de ces accusations, quelle est leur part de fiction et de réalité, et le sens qu'il est possible de leur donner, remarquons que les trois seules caricatures contre-révolutionnaires qui figurent un protestant ont choisi Rabaut Saint-Étienne; de nombreux pamphlets dénonçant le protestantisme le mettent en scène ou utilisent son nom. Pourquoi cette focalisation sur l'ancien pasteur de Nîmes?

(17) Voir Caricature 3 - Camus, Talleyrand, Rabaut-Saint-Etienne, la Religion. Présentée dans Claude LANGLOIS, La caricature contre-révolutionnaire, op.cit., p. 81.

(18) Le complot dans ce volume sert particulièrement les desseins républicains : «M. Necker avoit assisté (s'il ne l'avoit pas convoquée) à une assemblée tenue à Nismes, et qui scella l'union de la triple alliance. Il ne s'y étoit pas rendu seul : Lafayette, Dumouriez, Clavière, l'abbé Raynal et autres chefs d'émeute, encyclopédistes, philosophes et franc-maçons, calvinistes. Là furent repris le plan tant de fois conçu et avorté, dans les assemblées de Nismes en 1572 et 1575 ; d'Anduze de 1573 ; de Montauban de 1583 ; de La Rochelle, de 1621 et depuis encore : de mettre la France en république », Les Véritables auteurs, op. cit., p. 448.

(19) Secret échappé ou Dialogues entre M. Rabaud de Saint-Etienne, ministre Protestant, député à l'Assemblée nationale et deux Demoiselles Angloises, 1790. 


\section{Des usages d'une histoire plurielle}

Rabaut Saint-Étienne n'est pas le seul protestant qui ait participé à la vie politique au début de la Révolution : 17 sur les 1200 députés de la Constituante sont calvinistes ou luthériens, 20 des 745 de la Législative, 36 sur les 749 de la Convention ${ }^{20}$. Par exemple, Alba-Lasource, Barnave, Boissy d'Anglas, Cambon, Jay, Rühl, Marat, Jeanbon Saint-André, RabautPomier sont « de la religion», sans jamais s'identifier comme tels dans les débats ${ }^{21}$. Le Nîmois n'est pas le seul pasteur engagé dans la tribune politique et, d'ailleurs, au moment où sévissent le plus fortement les caricatures anti-révolutionnaires, c'est l'ancien pasteur de Castres, Alba-Lasource, qui fait partie de la Législative, aucun constituant ne pouvant s'y retrouver. Pourtant, Rabaut personnifie à lui seul le protestantisme, bien plus que Necker. Et ce qui semble le désigner à la vindicte publique est son statut de ministre : pour preuve, les caricatures qui, toutes trois, le représentent avec la robe noire à rabats blancs des pasteurs alors que David, dans son Jeu de Paume, le représente en « civil ${ }^{22}$. L'image de serviteur d'une Église domine dans les sources iconographiques. Elle demeure juste car il a été pasteur, mais elle « retarde » sur son état au moment où elles paraissent. Formé dans le Séminaire fondé par Antoine Court à Lausanne, Rabaut Saint-Étienne a débuté son ministère en 1765 , aux côtés de son père, Paul Rabaut ; il était alors un pasteur très écouté dans une région à forte identité réformée et le fils a acquis rapidement une stature digne des espérances du père ${ }^{23}$. Il ne prêche pourtant plus après son départ pour Paris et, une fois élu député pour la sénéchaussée de Nîmes et Beaucaire, il apparaît sur les registres comme «propriétaire foncier $»^{24}$. La présence de cette robe pastorale s'explique toutefois aisément dans les caricatures, par le

(20) Jacques PouJol, « Le changement d'image... », op. cit., p. 507-510.

(21) Jacques Poujol note toutefois trois cas d'identification au protestantisme à la tribune : celle du baron de Rathsamhausen, le 21 mai 1790, celle de Barnave lors des discussions autour de la Constitution civile du Clergé, le 4 janvier 1791 et, enfin, celle d'Alba-Lasource qui se définit comme « prêtre d'une autre religion » au moment des débats sur les prêtres non assermentés, le 25 août 1792 (Jacques Poujoul, « Le changement d'image... », op. cit., p. 506 et p. 509). Sur la culture protestante des députés voir également Timothy TACKETT, Par la volonté du peuple. Comment les députés de 1789 sont devenus révolutionnaires, Paris, Albin Michel, 1997, p. 65-66.

(22) Paul Romane-Musculus, « Histoire de la robe pastorale et du rabat », Bulletin de la Société de l'Histoire du protestantisme français, t. 115, 1969, p.307-328.

(23) Céline BoRELlo, « La profession de foi d'une dynastie pastorale du Désert : les Rabaut, des trois tomes du Jeune pommier à fruits précoces à l'exemplarité de saint Étienne » dans Didier POTON (dir.), Agir pour l'Église, Ministères et charges ecclésiastiques dans les églises réformées, Paris, Les Indes Savantes, 2014, p. 215-219.

(24) L'article IV de l'édit de Fontainebleau de 1787, dit « édit de tolérance », n'autorise pas les pasteurs à prendre ce titre dans les actes de la vie officielle. Les pasteurs n'ont toujours pas le droit d'exercer et il faut attendre la constitution du 3 septembre 1791 pour cela (Titre 1). 
genre même du support qui implique une simplification visuelle et quoi de plus évident que la robe pastorale pour désigner la Réforme ? Dans les textes son nom est souvent précédé de la mention «pasteur », « prédicant », «ministre $»^{25}$. De fait, il incarne bien plus facilement l'adversaire protestant dans les textes et les images polémiques, mieux que Necker, lui-même sans signe extérieur d'appartenance confessionnelle. Et d'ailleurs pour Calvin, n'est-ce pas les pasteurs qui sont le premier des quatre ministères qui structurent la communauté26? Les opposants aux huguenots ne s'y sont pas trompés en érigeant un pasteur comme symbole de l'ensemble des réformés.

Son appartenance au corps pastoral ne peut suffire à expliquer cette personnification, il n'est pas le seul pasteur à siéger dans les assemblées révolutionnaires. Un autre élément a sans doute joué : son aisance dans la prise de parole publique, en particulier au début de la Révolution. Dans la joute verbale des débats révolutionnaires cela demeure un atout. Jean Baubérot, dans sa préface à la réédition de la biographie de Rabaut Saint-Étienne, insiste sur ce point et, dans les Véritables auteurs de la Révolution française, les talents d'orateur de Rabaut sont rappelés comme signe de perfidie :

«Rabaud de Saint-Etienne réunissait à une grande pratique des hommes, un tact habile et beaucoup de facilité dans les affaires, cet esprit d'intrigue devenu talent du jour et une belle élocution. Dans l'assemblée soi-disant constituante, Rabaud de Saint-Etienne laissait l'abbé Mauri et Cazalès, et les orateurs du côté droit s'épuiser en raisonnement qui n'avoient pour eux que la vérité et l'avantage de l'état: il ménageait ses poumons et distribuait des raisons dont le poids, le titre et la valeur coulaient toujours à fond les arguments de ses adversaires et tamponnaient les oreilles de leurs auditeurs $\gg^{27}$.

(25) Il faut noter ici que les termes prédicant et ministre ne sont pas du même registre. S'ils définissent tous les deux celui qui prêche, le premier est péjoratif et était souvent utilisé au début du $\mathrm{XVIII}^{\mathrm{e}}$ siècle pour désigner ceux qui faisaient acte de prédication sans avoir toujours reçu la formation.

(26) Pour le réformateur de Genève, la direction de l'Église est constituée de quatre ministères : les pasteurs, les professeurs, les anciens et les diacres. Ce sont les Ordonnances de 1541 qui consacrent ces institutions, François WENDEL, Calvin, sources et évolution de sa pensée religieuse, Genève, Labor et fides, 1985 (réed.), p. 50.

(27) Les Véritables auteurs, op. cit., p. 21-22. Sur la mention de Jean Beaubérot, André DuPONT, Rabaut Saint-Étienne 1743-1793. Un protestant défenseur de la liberté religieuse, Genève, Labor et Fides, 1989, p. XIII. 
Vingt années de prédication dans le Désert languedocien lui ont donné une expérience réelle de l'art oratoire ${ }^{28}$. Bien qu'il ne soit pas un homme politique de premier plan, il se distingue comme seul pasteur parmi les dix-sept protestants de l'Assemblée constituante ${ }^{29}$. À côté de son passé de ministre et de sa qualité d'orateur, deux autres éléments éclairent cette cristallisation de la critique haineuse autour de Rabaut.

Tout d'abord, son origine nîmoise. Certains pamphlets proviennent de cette ville, comme Les Républiques fédératives ou encore Le Précis historique des massacres commis par les protestants sur les Catholiques ${ }^{30}$ dont l'auteur est le Nîmois et catholique Jacques-Marie Boyer-Brun, ce qui est déterminant pour comprendre les accusations et leur amplification ${ }^{31}$. En effet, Boyer-Brun est journaliste et part à Paris au moment des événements de Nîmes; il fait paraître des articles dans le Journal de la cour et de la ville ou Petit Gautier, puis devient rédacteur au Journal général de la France et fonde, en février 1792, Le Journal du Peuple, journaux de la presse contre-révolutionnaire et royaliste. Lorsqu'il lance la publication du périodique, Histoire des caricatures de la révolte des français, en avril 1792, il ne manque pas de reproduire les caricatures du Nîmois Rabaut, qu'il prétend connaître personnellement et qu'il déteste en tout point. Dans cette publication, ses commentaires expriment son aversion pour les protestants, qu'il désigne d'ailleurs comme les premiers à avoir utilisé la caricature «depuis Calvin jusqu'à présent », décrivant celle des Coups de Rabot ainsi :

«Ce monstre, moitié homme, moitié serpent, porte le rabat et la robe de prédicant. Au bout de sa longue queue, dont il veut cacher le dard, se

(28) Céline BoREllo, Du Désert au Royaume : parole publique et écriture protestante (1765-1788) - Édition critique du Vieux Cévenol et de sermons de Rabaut Saint-Étienne, Paris, Honoré Champion, 2013. Sur le pouvoir de la parole au sein de l'Assemblée, voir Timothy TACKETT, Par la volonté du peuple, op. cit., p. 214-222.

Alphonse Aulard consacre à Rabaut Saint-Étienne quelques pages dans son ouvrage sur l'éloquence parlementaire, remarquant qu'il fut un bon orateur mais de second plan même s'il se fit remarquer lors de son discours du 23 août 1789, voir François-Alphonse AULARD, L'éloquence parlementaire pendant la Révolution française. Les orateurs de l'Assemblée constituante, Paris, Hachette, 1882, p. 429-435.

(29) Timothy Tackett le place toutefois parmi les « participants les plus importants » dans sa présentation du Serment du Jeu de Paume de David, Timothy TACKETT, Par la volonté du peuple, op. cit., p. 215.

(30) Le Précis historiques des massacres commis par les protestants sur les Catholiques, dans les journées des 13, 14 \& 15 juin 1790, [Nîmes], 1790.

(31) Annie DUPRAT, « Le Regard d'un royaliste sur la Révolution : Jacques-Marie Boyer de Nîmes », $A H R F, \mathrm{n}^{\circ}$ 337, 2004, p. 21-29 ; id., « Jacques-Marie Boyer de Nîmes et l'image polémique », L'image à la lettre, Paris, Paris-Musées, 2005, p. 83-105. Voir également pour une présentation du personnage dans Claude LANGLOIS, La caricature contre-révolutionnaire, op. cit., p. 17. 
trouvent ces mots parfaitement caractéristiques : Je suis rampant comme le serpent, mais j' ai plus de venin que lui... $»^{32}$.

Cette thématique animalière, fréquente dans la caricature révolutionnaire, est présente aussi dans le Précis historique des massacres, qui décrit les protestants comme des « reptiles vénéneux nés de la dissolution publique $»^{33}$. Derrière cette figure pointe l'image du mauvais patriote, à la solde de puissances étrangères, qui trompe ceux qui l'écoutent, tel le Serpent biblique qui entraîne Adam et Ève dans la chute.

La chronologie de ces sources montre que la plupart d'entre elles paraissent entre mars et juin 1790 dans un contexte particulier pour la religion, pour Rabaut et pour Nîmes : la Constitution civile du clergé est débattue à l'Assemblée, la motion de Don Gerle pour que le catholicisme reste religion d'État demeure dans tous les esprits, Rabaut est, entre le 15 et le 28 mars 1790, président de la Constituante et, enfin, à Nîmes se préparent les élections municipales dont le résultat provoquera la « bagarre de Nîmes », précédemment mentionnée. Rabaut Saint-Étienne devient alors un symbole pour la région nîmoise, pour son présent et pour son passé. Et certains auteurs de ces sources anti-protestantes sont liés à ce territoire. Les caricatures sont plus tardives (novembre-décembre 1791) mais le décalage est habituel dans la caricature anti-révolutionnaire par rapport à l'image révolutionnaire. L'origine nîmoise de Rabaut demeure profondément inscrite dans la dénonciation et explique aussi la moindre présence de Necker dans ces discours. En effet, en juin 1765, à Nîmes, Jean-Paul Rabaut a été le premier fils de pasteur français à avoir été consacré par son père, lui-même pasteur de cette Église depuis 1741. Rabaut est le premier réformé à occuper un poste de président d'assemblée révolutionnaire, le seul de la Constituante. Suivent pour la Législative, Cambon et Laffon de Ladébat et, pour la Convention, huit présidents protestants dont le premier est encore Rabaut, en janvier 1793. Cette primauté dans bien des domaines fait de lui un huguenot aisément stigmatisable aux yeux des catholiques nîmois. Ceux-ci, au moment de sa nomination comme président de la Constituante, en mars 1790, et des élections locales de Nîmes, répondent d'ailleurs aux réformés, dans une courte chanson éclairant le lien entre Nîmes et Paris, autour de ce personnage. Alors que les protestants écrivent :

(32) CHAMPfLeury, Histoire de la caricature sous la République, l'Empire et la Restauration, Paris, Dentu, 1877, p. 186.

(33) Le précis historique des massacres commis par les Protestants, op.cit., p. 5. 
«Un seigneur [le baron de Marguerites], à force d'argent/S'est fait nommer maire céans/C'est ce qui nous désole (bis)/Mais à Paris un protestant [Rabaut Saint-Étienne]/On en a fait un président/C'est ce qui nous console (bis) ».

Les catholiques nîmois leur répondent :

«L'exécrable Assemblée a fait un président/Dont le choix à jamais déshonore la France./Au fauteuil est assis Rabaut le Prédicant,/Agent stipendié du l'huguenote engeance./Après avoir détruit et le trône et l'autel,/Poussé l'irréligion jusques au fanatisme,/Qui mieux que le héros de l'impur calvinisme/Aurait pu présider ces infâmes mortels ? ${ }^{34}$.

Les conflits confessionnels méridionaux opèrent ainsi un rôle certain dans la dénonciation anti-protestante nationale des années 1790-91.

Une dernière raison à la forte présence de Rabaut est qu'il incarne la figure parfaite de la compénétration du religieux et du politique avec une triple temporalité d'expression : celle du passé ancien du Désert, du passé plus récent de la reconnaissance civile acquise par l'édit de 1787 et du présent de la participation politique des protestants aux assemblées et activités révolutionnaires, dernier point difficilement acceptable pour certains catholiques ${ }^{35}$. En effet, Rabaut Saint-Étienne n'est pas n'importe quel pasteur. Son père, Paul Rabaut, avec l'aide d'Antoine Court, a joué un rôle fondamental dans la reconstitution des Églises durant les années 1740-1770. Dans la caricature Les Coups de Rabot, ce passé lointain du Désert est évoqué dans les cinq « $\mathrm{P}$ » de la table signifiant « pauvre peuple protestant, prends patience » : à maintes reprises son père prêcha des sermons d'exhortation à la patience - dans les années 1750 en particulier - rejetant toute forme de violence face aux attaques catholiques ${ }^{36}$. Le père, désigné comme «saint Ministre du Désert » dans Les Républiques

(34) Dans les rues de Nîmes a été affiché au même moment un placard intitulé L'infame assemblée nationale vient de mettre le comble à ses forfaits, elle a nommé un protestant pour la présider. Sur ces publications voir Armand LoDS, « Deux chansons sur Rabaut Saint-Etienne », La Révolution française, t. XLIV, p. 501.

(35) La résistance catholique a notamment pu être forte avec la Constitution civile du clergé qui permettait aux protestants de participer aux élections des évêques et curés constitutionnels (le corps électoral est celui des administrations locales).

(36) Albert MonOD, Les sermons de Paul Rabaut : pasteur du désert (1738-1785) : étude sur les manuscrits inédits de Paul Rabaut, suivie du texte de trois sermons annotés, Thèse complémentaire de doctorat ès-lettres présentée à la faculté des lettres de l'université de Paris, Impr. G. Carayol, 1917 ; Céline BORELLO, « Texte et contexte : la violence dans les sermons protestants du XVIII ${ }^{\mathrm{e}}$ siècle», Lucien FAGGION, Christophe REGINA (dir.), La violence. Regards croisés sur une réalité multiple, Paris, CNRS Éditions, 2010, p. 471-494. 
fédératives, y prend le premier la parole pour annoncer une prophétie républicaine ${ }^{37}$. À côté de ce passé du Désert, l'histoire plus proche explique également la forte présence de Rabaut Saint-Étienne : il a joué un rôle dans l'élaboration de l'Édit de 1787 qui légalise les protestants. Venu à Paris à la demande de La Fayette en 1786, il a été l'interlocuteur protestant des rédacteurs de l'édit de Versailles. Dans Les Coups de Rabot, l'évolution législative est rappelée, au bas de l'établi, par l'inscription « les tems ont bien changé ». Enfin, l'histoire du temps présent de la Révolution explique son exposition dans cette littérature polémique : il prend la parole au sein des assemblées lors des discussions et débats importants ${ }^{38}$. Se déploie sur différentes temporalités, une mise en perspective historique où textes et images se répondent, non seulement sur l'histoire du passé protestant dans sa globalité - et dans la ville de Nîmes tout particulièrement - mais aussi sur l'histoire personnelle de ce pasteur. En ce sens, durant la Révolution, Rabaut Saint-Étienne devient l'homme de la situation pour diaboliser les protestants, des origines de la Réformation à la période de l'écriture de ces textes, et alors que les histoires nîmoise ou parisienne, nationale ou individuelle, s'entremêlent et insufflent une dimension plus forte encore à l'action protestante ainsi dénigrée.

\section{L'entrée en politique des protestants par la calomnie révolutionnaire}

La confrontation entre ces accusations et la question du « changement d'image » des protestants pendant la Révolution - la détérioration de cette image - doit dès lors être reposée. Tout d'abord, d'après Jacques Poujol «l'image protestante (positive) atteignit son zénith en décembre 1791 avec l'Almanach du Père Gérard» de Collot d'Herbois. Ensuite, un changement d'image s'opérerait dans une dénonciation des huguenots

(37) « Mon fils fera échec \& mat au Roi \& à l'aide de quelques fous \& de quelques cavaliers \& fantassins, il renversera les tours élevées par l'aristocratie, \& fera sauter les pions du Roi, enfermera \& fera sauter le Roi lui-même. Génie de Calvin, de Milton \& de Jurieu, inspirez mon fils ; transmettez-lui la haine des Rois, dont l'insolence est armée d'un sceptre sanguinaire, dont le pouvoir n'est qu'une licence féroce \& qui insultent au pauvre peuple », Les Républiques fédératives, op. cit., p. 4 et 5

(38) Outre le discours de Rabaut Saint-Étienne du 23 août 1789, ont été imprimées ses prises de paroles suivantes : Question de droit public : doit-on recueillir les voix dans les États-généraux, par ordres ou par têtes de délibérans ?, 1789 ; Réflexions sur la division nouvelle du Royaume et sur les privilèges et les assemblées des provinces d'États, Paris, Baudouin, 1789 ; Projet du préliminaire de la constitution française, Versailles, Baudouin, 1789 ; Opinion de M. Rabaut sur la discussion ouverte dans l'Assemblée nationale au sujet des mouvements de plusieurs princes de l'Europe, 28 juillet 1790, Paris, Impr. nationale, 1790 ; Rapport sur l'organisation de la force publique, fait au nom du comité de constitution \& du comité militaire, le 21 novembre 1790, Paris, Impr. nationale, 1790 ; Projet d'éducation nationale du 21 décembre 1792, Paris, Impr. nationale, 1792/1793. 
qui traduirait une « détérioration systématique de l'image protestante $»^{39}$. Certes, dans l'Almanach cité, les protestants sont nommés "frères ", tout comme dans le Serment du jeu de Paume, Rabaut est présenté en rassembleur. Mais, à la même période, plusieurs caricatures l'invectivent très violemment et, à travers lui, l'ensemble de la communauté réformée. Bien plus, certains pamphlets, comme Les Républiques fédératives, sont antérieurs à cette date et voici comment le pseudo-frère de Rabaut présente l'action protestante dans un geste de rébellion généralisée :

« armés comme autrefois de torches $\&$ de poignards, nous parcourrons, nous saccagerons, nous dévasterons le Royaume, nous ensevelirons sous les ruines du Trône, des Tribunaux, des Églises \& des Autels, la Monarchie, l'Autorité, la Religion ; nous ferons tomber sur la génération présente, tous les malheurs dont les générations passées ont accablé nos pères; nous baignerons dans le sang; nous établirons sur des morceaux de cendres $\&$ des cadavres ; ensuite, $\&$ de nos mains ensanglantées, nous élèverons l'édifice du bonheur public $\&$ de la régénération universelle $»^{40}$.

D'autres pamphlets antérieurs à 1791 stigmatisent les protestants en usant des mêmes procédés ${ }^{41}$. Il y a continuité chronologique dans la dénonciation catholique du rôle des protestants durant les premières années

(39) Jacques PoujoL, « Le changement d'image des protestants », op. cit., p. 521 et 539.

(40) Les Républiques fédératives, op. cit., p. 37.

(41) En plus de ceux évoqués précédemment, les textes stigmatisant les protestants sont les suivants : Discours à lire au Conseil en présence du Roi par un ministre patriote sur le projet d'accorder l'État civil aux protestants, 1787, s.1., [texte attribué à l'abbé Bonnaud, à l'abbé Lenfant ou au Père Parfait, réedité en novembre 1791] ; Pierre Romain, Pierre Romain aux catholiques de Nîmes, 1789 ; Charles SINCÈRE, Charles Sincère à Pierre Romain, 1789 ; Jean-Baptiste DuVOISIN, La France chrétienne, juste et vraiment libre, 1789, s.1. ; Martin-François THIÉBAULT, Discussion de cette proposition de M. de Custine : La liberté de l'exercice public de toutes les religions doit être prononcée dans l'Assemblée nationale, Metz, Collignon, 1789 ; Délibération des citoyens catholiques de la ville de Nîmes prise le 20 avril 1790, s.d., s.l. ; Gaspard JEAUFFRET, De la religion à l'Assemblée nationale. Discours philosophique et politique où l'on établit les principaux caractères qu'il importe d'assigner au système religieux pour le réunir au système politique dans une même constitution, Paris, Le Clere-Frouillé, 1790 ; Dénonciation aux Français catholiques, des moyens employés par l'Assemblée nationale pour détruire en France la Religion catholique, Londres, Paris, 1791; Claude Felix MonTJoIE, L'ami du Roi des Français de l'Ordre et surtout de la Vérité ou Histoire de la révolution en France et de l'Assemblée nationale, Paris, 1791 ; Lettre de M. L'Evêque d'Uzès dont une copie a été lue à l'assemblée Nationale et dénoncée comme incendiaire, Paris, Crapart, 1791 ; Conjuration contre la religion catholique et les souverains dont le projet, conçu en France, doit s'exécuter dans l'Univers entier, Paris, Crapart, 1792 ; Les Français devenus protestants sans le savoir ou Parallèle de la religion protestante et de la nouvelle religion de France. En réfutation du Concordat de M. Gratien évêque du département de la Seine inférieure et Métropolitain des Côtes de la manche, Pairs, Lallemand, 1792 ; La grande question ou les jureurs devenus huguenots, Paris, seconde édition 1792 ; VAUVILLIERS, La doctrine des théologiens ou seconde partie du témoignage de la raison et de la foi contenant le parallèle de la doctrine de M. Larriere avec celle des Protestants, \& la réfutation de la suite de son Préservatif contre le schisme, Paris, Desaint, 1792. 
de la Révolution, et si certaines voix prônent la réconciliation elles sont simultanées au dénigrement, plutôt qu'antérieures : le changement d'image est difficilement perceptible.

Une seconde remarque est que cette accusation protestante ne produit aucun discours réellement neuf et, en ce sens, il est délicat de postuler 《une détérioration systématique de l'image protestante $»^{42}$. Les reproches, à partir de la figure de Rabaut Saint-Étienne, demeurent traditionnels. La critique de la dérégulation sociale, de la rébellion protestante n'est pas contemporaine de la Révolution et a été réactivée par la propagande catholique dès la guerre des Camisards au début du XVIII ${ }^{\mathrm{e}}$ siècle ; tout comme l'accusation de républicanisme ou bien encore la volonté de détruire l'Église catholique pour imposer la religion réformée qui ne représentent pas un discours nouveau. Ces griefs sont déjà présents dans la littérature d'Ancien Régime. Ainsi dans la Lettre de M. L'évêque d'Agen à M. Le contrôleur général contre la tolérance des huguenots dans le royaume, paru en 1751, s'expriment les mêmes dangers pour la monarchie :

«Si l'on veut considérer de près les principes du calvinisme, on verra que non seulement ils sont opposés à toutes les religions quelles qu'elles soient, mais de plus qu'ils sont ennemis des Rois et opposés à la monarchie, c'est un caractère qui lui est propre et qui doit le faire détester par-dessus toutes les autres hérésies [...]À peine les calvinistes se sont-ils montrés dans le monde, qu'on a vu tous les royaumes ébranlés par leurs maximes séditieuses et par leurs armes $\gg^{43}$.

Suit alors un tour d'Europe pour prouver cette relation : Flandre, Hollande, Écosse et Angleterre. Reprenant la corrélation entre protestantisme et républicanisme développée par Montesquieu ou Voltaire au milieu du XVIII ${ }^{\mathrm{e}}$ siècle, le Discours à lire au Conseil en présence du Roi par un ministre patriote sur le projet d'accorder l'Etat civil aux protestants, réactive en 1787 l'idée de l'esprit républicain des huguenots ${ }^{44}$. Ce texte

(42) Jacques PouJoL, « Le changement d'image des protestants », op. cit., p. 539

(43) Lettre de M. L'évêque d'Agen à M. Le contrôleur général contre la tolérance des huguenots dans le royaume, transcrite dans Otto SELLES, Le Patriote français et impartial, Paris, Honoré Champion, 2002, p. 12.

(44) «La Rochelle était le boulevard de la révolte soufflée par l'hérésie. C'est là que le plan, projeté longtemps auparavant, de changer la Monarchie Française en une République, qui serait administrée par les Calvinistes eut enfin son exécution. Tout le Roiaume devoit être partagé en huit cercles : c'étoit une analogie avec les cercles d'Allemagne. [On avoit dressé un grand règlement de quarante-sept articles, que devoit observer les Commandants, sous l'autorité souveraine de l'Assemblée séante à La Rochelle. Ils devoient sous les ordres d'un prince étranger, avoir une puissance égale, sans s'arrêter aux Princes de Sang]. Cette pièce, que nous ont conservés les journaux du temps est si curieuse 
insiste également sur la dérégulation sociale portée par le protestantisme, de la même manière que les pamphlets révolutionnaires postérieurs ${ }^{45}$. La seule nouveauté dans l'argumentaire anti-protestant de la Révolution est la collusion avec les francs-maçons et les philosophes. Elle relève spécifiquement du contexte de la fin du XVIII ${ }^{\mathrm{e}}$ siècle et se retrouve notamment dans les journaux. Ainsi dans la Gazette de Paris, le 31 octobre 1790, le journaliste royaliste Du Rozoi mêle-t-il républicanisme et connivence protestante $^{46}$. La complicité est aussi entre philosophes, jansénistes et protestants comme le montre la caricature Camus, Talleyrand, RabautSaint-Etienne, la Religion. Mais s'agit-il ici d'autre chose que du thème ancien du complot contre l'Église catholique ${ }^{47}$ ? Dans la dénonciation des protestants sous la Révolution sont repris des thèmes classiques de la propagande catholique des siècles antérieurs : aux yeux des polémistes de cette religion, alors souvent du côté contre-révolutionnaire et royaliste, l'image des huguenots reste négative, elle n'est pas détériorée.

La stigmatisation ne peut toutefois se couper du contexte dans lequel elle se situe et c'est là, sans doute, qu'il faut lui rendre sa signification et sa profondeur. Il y a indéniablement de la calomnie dans les propos

et si importante, qu'il est nécessaire de la mettre sous les yeux de V.M.. Ce monument doit vous convaincre, SIRE, que l'esprit du calvinisme est essentiellement républicain, et dès lors inconciliable avec la monarchie française », Discours à lire au Conseil en présence du Roi par un ministre patriote, op. cit., p. 59-60. La corrélation entre républicanisme et protestantisme se retrouve dans le livre 24 de L'Esprit des Lois de Montesquieu (1748) dont le chapitre V s'intitule «Que la religion catholique convient mieux à une monarchie, \& que la protestante s'accommode mieux d'une république ».

(45) La présentation de la révocation de l'édit de Nantes donne l'occasion à l'auteur de montrer les dangers que représentent les huguenots pour l'ordre public et social : « Ainsi fut anéanti dans ce Royaume le plus furieux, le plus terrible de tous les ennemis que la France ait jamais eu ; celui qui l'a désolée par le fer et par le feu, qui l'a livrée à l'avarice \& à l'ambition des étrangers, qui l'a réduite aux dernières extrémités, par la fureur des guerres civiles, par des révoltes tant de fois réitérées, par tous les horribles excès de la rage de l'impiété ; qui a fait la guerre à six Rois de France (I : note : François II, Charles IX, Henri III, Henri IV, Louis XIII, Louis XIV), \& leur a livré quatre batailles rangées », Discours à lire au Conseil en présence du Roi par un ministre patriote, op. cit., p.77

(46) «Il ne faut jamais perdre de vue cette faveur distinguée accordée au Protestantisme, cette chaîne qui unit le système protestant au système républicain, depuis les confins de l'Allemagne, jusqu'à ceux du midi de la France. En Languedoc, dans le Quercy, dans le Béarn, en Alsace, même prédilection pour les Enfants de Luther et de Calvin. Successivement les dogmes de ces deux prétendus réformateurs (Luther et Calvin), embellis du vernis de cette Philosophie moderne, à laquelle s'est joint l'esprit d'un autre régime, qu'il suffit de nommer pour le faire connaître, détruisent les Loix des Empires, asservissent l'Opinion et préparent des Révolutions qui changeront, si les Souverains ne s'y opposent, la face entière de l'Europe. Ce régime, si favorable au système Républicain, analogue aux succès des Missionnaires de la Propagande, c'est celui des Francs-Maçons », cité par Jean-Paul BERTAUD, Les amis du roi, Journaux et journalistes en France de 1789 à 1992, Paris, Perrin, 1984, p. 69.

(47) Dans ce panel des ennemis de la religion et des instigateurs de la Révolution, les juifs ne sont pas oubliés. La collusion entre protestantisme et judaïsme apparaît dans la Rocambole des Journaux où Rabaut explique aux calvinistes : « Si vous avez besoin d'aides, adressez-vous aux juifs, ils vous seconderont parfaitement », Id., p. 67. 
évoqués et dans ces images exposées ${ }^{48}$. Ainsi les protestants français des années antérieures à 1792 ne peuvent être suspectés de républicanisme, la loyauté à l'égard du monarque a été constante et réitérée à plusieurs reprises dans des sermons, des écrits, des mémoires, des adresses durant tout le XVIII ${ }^{\mathrm{e}}$ siècle $^{49}$. Rabaut lui-même, dans son Almanach historique de la Révolution française paru en 1792, indique que la France n'est pas mûre pour la République qui ne conviendrait pas à ce pays trop vaste et trop peuplés ${ }^{6}$. Mais ces mots et ces images ${ }^{51}$ participent d'un discours qui se durcit, qui devient performatif comme l'ont montré Jean-Paul Bertaud, Jacques Guilhaumou ou Pierre Serna ${ }^{52}$. C'est d'une «radicalisation», plus que d'une détérioration dont il s'agit dans ce dossier protestant. Radicalisation qui trouve son actualisation dans l'histoire révolutionnaire en train de se faire, tout en puisant ses sources dans la polémique traditionnelle d'Ancien Régime.

Actualisation, par exemple, lorsque Rabaut prend la parole, le 21 novembre 1790, sur l'organisation de la force publique : sa position sur le renforcement de la maréchaussée comme « force publique intérieure », sur l'augmentation des effectifs de la garde nationale devient, dans un pamphlet paru au même moment (Copie d'une lettre de $M^{* * *}$, ministre calviniste des Cévennes, à $M$. Ra. De S. Et.) une preuve supplémentaire que les protestants constituent alors une armée à leur solde ${ }^{53}$. Mais les fondements de ces discours sont antérieurs aux événements révolutionnaires et l'argumentation rejoint la calomnie qui a touché les protestants avant même 1789. Cependant, on mesure l'impact du discours en ces temps de Révolution où tout est bouleversement, où tout devient potentiellement possible. L'amplification des prises de position grâce à la presse et au rayonnement de l'opinion

(48) Charles Walton, Policing Public Opinion in the French Revolution: the Culture of Calumny and the Problem of Free Speech, Oxford, Oxford University Press, 2009.

(49) Parmi les dernières publications voir LA BEAUMELLE, Deux Traités sur la tolérance. L'Asiatique tolérant (1748). Requête des protestants français au roi (1763), Édition critique par Hubert Bost, Paris, Honoré Champion, 2012.

(50) RABAUT SAINT-ÉTIENNE, Almanach historique de la Révolution française pour l'année 1792, Paris, Onfroy, 1792, p. 279.

(51) Le Journal de la cour et de la ville mentionne à propos de la caricature Les Brigands d'Avignon : cette «nouvelle caricature qui ne fera rire personne », Claude LANGLOIS, La caricature contre-révolutionnaire, op. cit., p. 71.

(52) Jean-Paul BERTAUD, La presse et le pouvoir de Louis XIII à Napoléon $1^{\text {er }}$, Paris, Perrin, 2000 ; Jacques GuILHAUMOU, « La "guerre de mots". On dit, nouvelles et dialogues dans la presse révolutionnaire (1791-1793) », Michel BIARD, Annie CRÉPIN, Bernard GAINOT (dir.), La plume et le sabre. Hommages offerts à Jean-Paul Bertaud, Paris, Publication de la Sorbonne, 2002, p. 101-110. ; Pierre SERNA, « Radicalités et modérations, postures, modèles, théories. Naissance du cadre politique contemporain », AHRF, 2009/3, p. 3-20.

(53) RABAUT SAINT-ÉTIENNE, Rapport sur l'organisation de la force publique, op.cit. 
publique décuple la portée d'un discours anti-protestant qui croise l'histoire de la Révolution. Un autre pasteur, Jeanbon Saint-André, note ainsi dans une de ses lettres de 1792, adressée au consistoire de son Église :

« Depuis le commencement de la Révolution jusqu'à présent, les malintentionnés n'ont cessé de rejeter sur les protestants le blâme de tout ce qui s'est fait ; le Journal général de France, le Mercure et tous les journalistes aux gages de l'aristocratie répètent jusqu'à satiété cette inculpation. Ils la répéteront encore et elle ne sera abandonnée que quand l'espoir d'une contre-révolution sera entièrement dissipé ${ }^{54}$.

L'argumentaire anti-huguenot rejoint alors le fantasme qui se transforme en dénigrement, appelant à la condamnation d'un individu ou d'un groupe détesté. Le précis historique des massacres commis par les Protestants se termine par la prophétie de la fin de Rabaut :

«Réjouis toi $\mathrm{R}^{* * * * *}$, tes desseins affreux ont réussi, tes satélites triomphent, leurs horreurs sont justifiés. [...] Mais tremble scélérat, quelle que soit ta prospérité actuelle, elle aura un terme. Rarement le crime reste impuni ; les tiens sont d'une nature à provoquer toutes les vengeances, à rendre ta mémoire exécrable, \& à cette nation qui t'honore de sa confiance, $\&$ à l'humanité entière dont tu es l'opprobre $»^{55}$.

La mise à mort est symboliquement portée par les mots mais elle se veut aussi forte que la haine de Boyer-Brun envers les protestants. Si, chez cet auteur, l'altérité religieuse et l'origine nîmoise fondent le propos, pour les lecteurs et les contemporains ces attaques doivent être d'interprétation politique. Et elles marquent en tout cas, par leur férocité et leur violence, la véritable entrée des protestants dans la sphère politique française. D'ailleurs Rabaut Saint-Étienne est parfaitement conscient de la nature politique de ces attaques ad hominem. Dans une lettre du 29 août 1791, il écrit : « je ne suis pas surpris des calomnies qu'on répand contre moi, c'est la portion des hommes publics dans le grand festin de la Révolution $»^{56}$.

(54) Lettre de Jeanbon Saint-André à « MM le Pasteur et anciens de Montauban », 15 mai 1792, cité dans Léon LÉVY, Quelques recherches sur Jeanbon Saint-André, Paris, Imprimerie de la Cour d'Appel, 1893, p. 13.

(55) Le précis historique des massacres commis par les Protestants, op. cit., p. 43-44. Les « satellites » désignent dans le texte, en note, « les milices des Cevennes \& de la Vaunage ».

(56) François RouviÈRE, «Quatre lettres inédites de Rabaut-Saint-Étienne, 1789-1791 : Documents pour servir à l'histoire de la Révolution française », Bulletin de la Société de l'Histoire du protestantisme français, t. 34, 1885, p. 225. 
Malgré la salve des mots contre celui qui fut ministre protestant, nuançons l'articulation entre le discours anti-calviniste et la dénonciation politique en rappelant que ce n'est pas son appartenance à la Réforme qui le fait déclarer traître à la patrie, le 28 juillet 1793 , mais sa position de girondin au sein de l'Assemblée. Pourtant un commentaire de Robespierre, le jour de l'exécution de Rabaut Saint-Étienne ${ }^{57}$, montre combien l'amalgame religion-politique demeure une modalité d'expression aisée et stigmatisante ${ }^{58}$. La radicalisation générale du discours qui devient, sous la Révolution française, d'une extrême violence condamne Rabaut et d'autres avec lui - à monter sur l'échafaud en $1793^{59}$. L'altérité religieuse et sa dénonciation, l'anti-protestantisme puisent leurs racines dans les vieilles haines et les discours traditionnels. La proposition de dégradation de l'image protestante disparait avec celle de son actualisation dans les événements politiques du moment.

(57) Sur les dernières semaines de la vie de Rabaut Saint-Étienne voir Armand LoDS, « Rabaut Saint-Étienne. Sa mise hors la loi - Son arrestation - Sa mort (14 novembre 1793, 5 décembre 1793) », Bulletin de la Société de l'Histoire du protestantisme français, t. 42, 1893, p. 510-541.

(58) «Les puissances étrangères ont dit à leurs émissaires : vous pouvez tout avec le peuple français, il ne faut que vous en emparer ; il est sensible, il aime la liberté ; sous cet appât cachez nos projets, vous les exécuterez. Savez-vous ce qui me confirme l'existence de cette conspiration ? C'est la découverte du traître que vous avez mis hors la loi. Vous auriez cru ce monstre traînant loin de vous sa honte et ses crimes; eh bien ! ce Rabaut, ce ministre protestant était à Paris, bravant la puissance nationale sous les yeux mêmes des représentants du peuple, et d'ici secouant les brandons de la guerre civile, et attisant le fanatisme dans les départements », Séance du 5 décembre 1793, cité par Armand LODS, « Rabaut Saint-Étienne. Sa mise hors la loi... », op. cit., p. 539.

La suspicion d'être à la solde des puissances étrangères touche également certains catholiques comme Claude Fauchet (Rita HERMON-BELOT, «L'abbé Fauchet », François FurET et Mona OzOUF (dir.), La Gironde et les Girondins, Paris, Payot, 1991 p. 329-349) ou Adrien Lamourette (Caroline BLANCChopelin, De l'apologétique à l'Église constitutionnelle : Adrien Lamourette (1742-1794), Paris, Honoré Champion, 2009).

(59) Y compris dans les discours intimes comme dans le journal du Marquis de Bombelles, réfugié dans la principauté de Saint-Gall et qui écrit à l'année 1793 : « Le 19 décembre - [...] Les Moniteurs arrivés aujourd'hui nous ont confirmé la mort de Rabaut-Saint-Etienne ; ce prédicant qui crut pouvoir élever son église protestante sur les ruines de la catholicité a porté sa tête sur l'échafaud où les autres factieux qui dominent aujourd'hui trouveront aussi la fin de leur horrible existence », Jean GRASSIAN, Frans DURIF et Jeannine CHARON-BORDAS (texte établi, présenté et annoté par), Marquis de Bombelles, Journal, publié sous les auspices du comte George Clam Martinic, tome 4, 1793-1795, Genève, Librairie Droz, 1998, p. 151. 


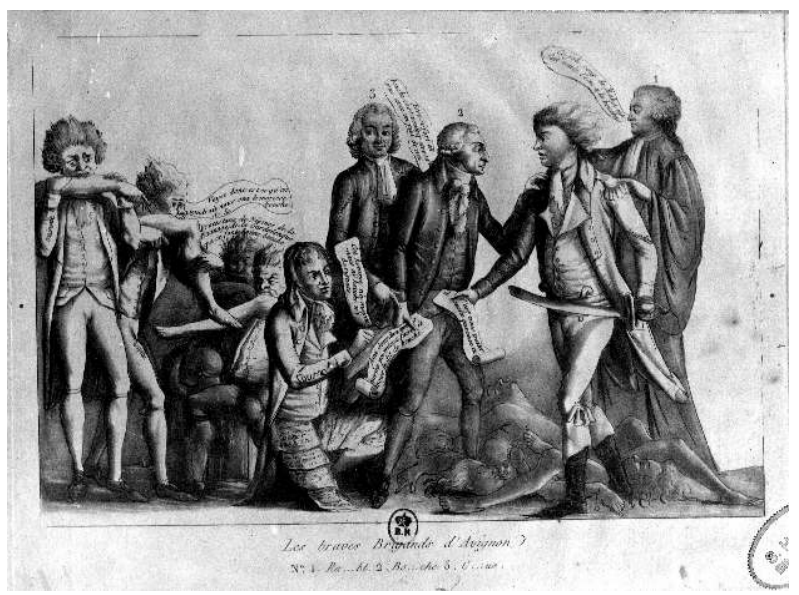

Caricature 1 - Les braves brigands d'Avignon (ㄷ Société de l'histoire du protestantisme français, Paris)

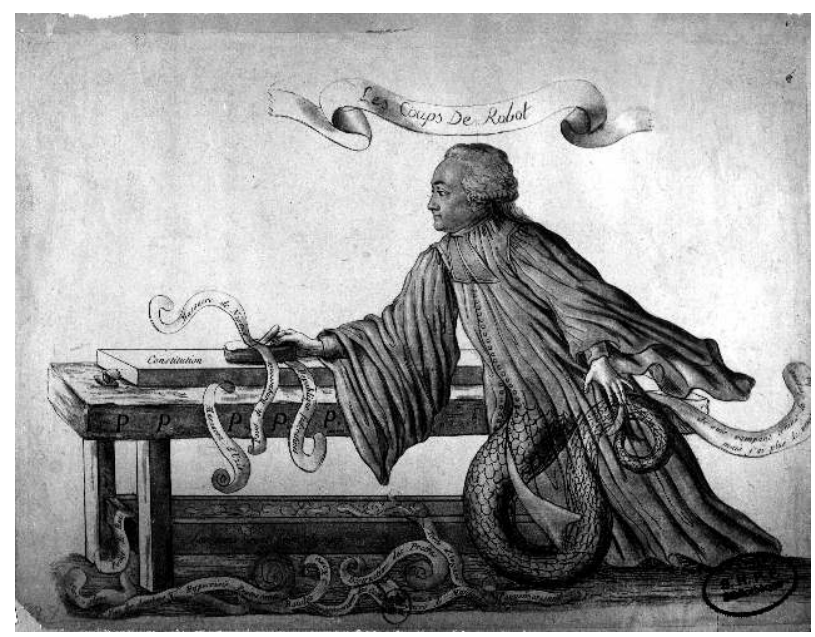

Caricature 2 - Les coups de Rabot (C) Société de l'histoire du protestantisme français, Paris) 


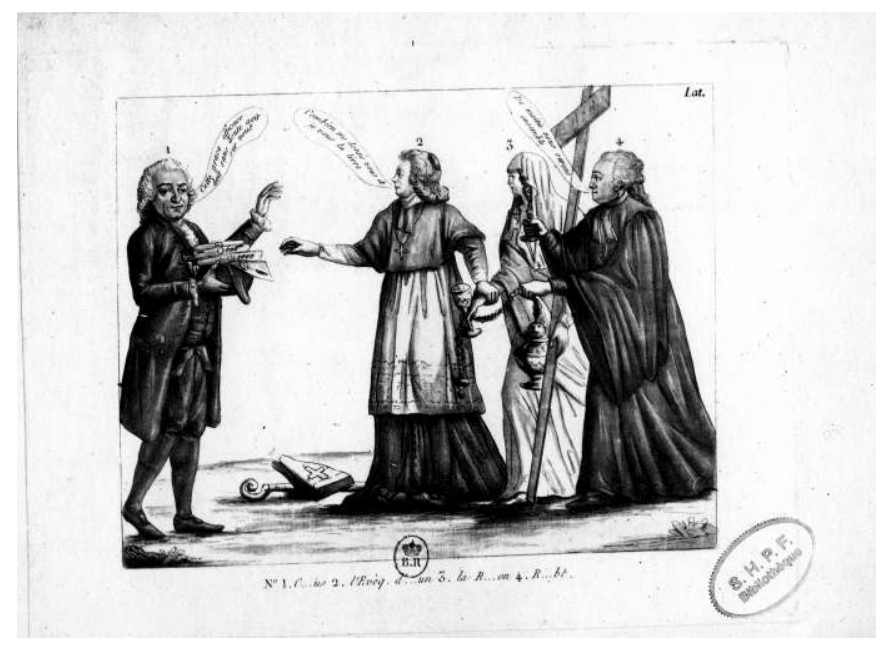

Caricature 3 - Camus, Talleyrand, Rabaut-Saint-Etienne, la Religion (C) Société de l'histoire du protestantisme français, Paris)

Céline BORELLO Université de Haute-Alsace, CRESAT celine.borello@uha.fr 